\section{Drug Dependent Stress and Heat Shock Protein Response}

Keywords: Heat shock protein; Alcohol; Drug addiction

Received: May 27, 2016; Accepted: June 08, 2016; Published: June 15, 2016

\section{Stress Response}

Stress induces radicals and this process triggers several biochemical pathways but most essential problem is the damage caused by the radicals on lipids, proteins, and nucleic acids. To perform biochemical functions, these macromolecules must be working properly. Proteins must be correctly folded so that they can regulate pathways and transmit signals. Under stress, alteration in cellular environment cause proteins to lose their structure. Then, the proteins either lose or alter their function. To scavenge radicals and to chaperone substrate proteins, a set of mechanic proteins are employed.

\section{Mechanics; Heat Shock Proteins}

Heat Shock Proteins (Hsps) are conserved universally from basic to most complex organism and serve to respond to cellular stress by unique biochemical mechanism. Hsps are chaperone cellular proteins by maintaining their native folded form so that they can perform their biochemical function promptly [1]

Hsps are classified according to their molecular weight; Hsp27, Hsp40, Hsp60, Hsp70, Hsp90, Hsp100, small Hsps. Each Hsp has redundant forms and the isoforms can be found at different cellular compartments i.e. cytosol, mitochondria, ER. Hsps are expressed as constitutively or inductively. Further, Hsps cooperate and coordinate with each other to form a variety of biochemical functions [2].

Details of Hsp network are still elusive and research is underway to understand the molecular mechanism of this network. Hsps are induced by several factors including infectious agents, heat, parasites, fungi, ischemia, malignancy, and oxidative stress as well as drugs [1].

Induction of Hsps indicates stress conditions in the metabolism and Hsps protect cells. Therefore, several metabolic diseases are associated with Hsp response. The aim of this paper is to review drug dependent stress and related biochemical events reported in the literature.

\section{Drug Dependent Stress}

Novel techniques help researchers to investigate molecular mechanisms of the drugs and Hsps are involved in stress related

\section{Lutfi Tutar ${ }^{1}$ Kubra Acikalin Coskun² and Yusuf Tutar ${ }^{2}$}

1 Kahramanmaraş Sutcu İmam University, Graduate School of Natural and Applied Sciences, Department of Biology, Kahramanmaraş, Turkey

2 Cumhuriyet University, Faculty of Pharmacy, Department of Basic Sciences, Division of Biochemistry, Sivas, Turkey

\section{Corresponding author: Yusuf Tutar}

ytutar@cumhuriyet.edu.tr and ytutar@yahoo.com

Professor at Cumhuriyet University, Faculty of Pharmacy, Basic Sciences Department, Division of Biochemistry, 58140, Sivas, Turkey.

Tel: +90 3462191010 Ext 3907

Fax: +903462191634

Citation: Tutar L, Coskun KA, Tutar Y. Drug Dependent Stress and Heat Shock Protein Response. J Drug Abuse. 2016, 2:2.

biochemical pathways. This leads researchers to consider Hsp related pathways when developing new drugs. Certain types of Hsp inhibition may lead cells to go apoptosis but inhibition of Hsp may not be beneficial for patients with neurodegenerative disease since some Hsps inhibit protein aggregation. Protein aggregation is the main cause of neurodegenerative diseases and blocking Hsps may augment aggregation and loss of treatment response. Thus, it is a dilemma for a cancer patient with a neurodegenerative disease. Several treatment regimens may be performed for such a patient but it is a double-edged sword.

Since Hsps cooperate and coordinate with each other, inhibiting specific pathways must be neatly chosen, otherwise several side effects of the drug treatment may change life quality of the patient. Several drug related cases of Hsp involvement is reported in the literature.

MDMA (3,4-Methylenedioxymethamphetamine) is an addictive drug and known as ecstasy. MDMA consumption has been associated with neurological and psychiatric disorders and induces morphological alterations in cortical neurons [3]. 
Amphetamine treatment may lead to elevated body temperatures and as a consequence Hsp70 expression levels increases at liver and brain tissues [4]. This elevation may result protection against MDMA toxicity as reported by a different research group [5]. Further, Hsp27 and Hsp70 upregulation studies in rat liver along with amphetamine pretreatment showed protection against acute dose of drugs. And the reason behind this event is probably lies in the activation of Hsp response [6].

Hsp90 plays a key role in metabolism and 2 to 3 percent of the proteins expressed in the proteome consist of Hsp90. Hsp90 role in alcoholic livers was investigated and inhibition of this protein by 17-DMAG (17-dimethylamino-ethylamino-17demethoxygeldanamycin) prevented liver injury. The inhibition decreased steatosis and pro-inflammatory cytokines. Hsp90 helps cell survival and Hsp90 inhibitor perturbs this function and damaged cells may go to apoptosis during alleviation of the liver. Therefore, Hsp90 is an essential target for alcoholic cirrhosis [7].

Moderate alcohol exposure impairs immune response and Heat Shock Factor 1 and Hsp70 inhibits TLR4/MyD88 pathway. Thus, in alcohol abuse treatment Hsps may be critical for clinical treatment [8]. Alcohol stress may provoke similar response as heat shock and activates Hsps to protect membranes and other cellular parts against alcohol stress [9].

Research on the effect of morphine on the regulation of protein degradation in the brain to elucidate involved proteins suggest that morphine induces Hsp70 expression in the striatum. This is most likely Hsp70 function in ubiquitin proteasome system to degrade the proteins. Hsp70 is also involved in protein aggregation pathway. If $\mathrm{Hsp} 70$ cannot refold or dissolve protein aggregates, then it sends the substrate proteins-aggregates to proteasome for degradation. The morphine induced Hsp70 expression research is not an exception $[1,10]$.

Hsp70 role in drug addiction was studied to understand the adaptation of neural networks by drugs of abuse. The research reports a correlation between behavioral sensitization to morphine and Hsp70 as evidenced by Hsp70 inhibition of Hsp70. The study suggested that Hsp70 is a target for adaptations with relevance to addiction [11]

Morphine withdrawal induces activation of hypothalamopituitary-adrenocortical axis and alters Hsp27 expression and suggest a role for Hsps in $\beta$-adrenoceptors and extracellular signal-regulated protein kinase pathways upon morphine related stress [12]. As expected, Hsps may be induced by stress but which Hsps are induced upon different stressors is yet be determined.

\section{Conclusion}

Drug treatment or drug addiction are two adverse concepts but both process induces stress albeit differently. The stress involves $\mathrm{Hsp}$ response and inhibition-induction of Hsp family may provide benefit to the patient and increase the life quality of the patient as well. Determining molecular mechanism behind drug dependent stress provides valuable information to drug developing researchers. A different subset of Hsps are employed for each and adverse conditions. Therefore, inhibition or induction conditions as well as Hsps involved in a pathway make researchers to design specific drugs. For this purpose, my group determines key Hsps involved in specific occasions by microarrays or transcriptome analysis. The experiments reveal key Hsps involved in the pathway for innovative drug development. We developed drug candidates for breast cancer and prion (a neurodegenerative disorder) and in each case a different set of Hsps involved. However, the designed compounds are inducers in prion and inhibitors in cancer. In both diseases overlapping Hsps are observed but different strategies employed for the treatment.

In summary, stress dependent Hsps must be determined for each disease to develop specific drugs in the treatment either by inhibiting or inducing key Hsp proteins. 


\section{References}

1 Tutar L, Tutar Y (2010) Heat shock proteins; an overview. Curr Pharm Biotechnol 11: 216-222.

2 Tutar $Y$, Song $Y$, Masison DC (2006) Primate chaperones Hsc70 (constitutive) and Hsp70 (induced) differ functionally in supporting growth and prion propagation in Saccharomyces cerevisiae. Genetics 172: 851-861.

3 Ruscher K, Fernandes E, Capela JP, Bastos Mde L, Wieloch T, et al. (2011) Effect of 3,4-methylenedioxyamphetamine on dendritic spine dynamics in rat neocortical neurons--involvement of heat shock protein 27. Brain Res 1370: 43-52.

4 Nowak TS Jr (1988) Effects of amphetamine on protein synthesis and energy metabolism in mouse brain: Role of drug-induced hyperthermia. J Neurochem 50: 285-294.

5 Escobedo I, Peraile I, Orio L, Colado MI, O'Shea E (2007) Evidence for a role of $\mathrm{Hsp} 70$ in the neuroprotection induced by heat shock pretreatment against 3,4-methylenedioxymethamphetamine toxicity in rat brain. J Neurochem 5: 1272-1283.

6 Salminen WF Jr, Voellmy R, Roberts SM (1997) Protection against hepatotoxicity by a single dose of amphetamine: the potential role of heat shock protein induction. Toxicol Appl Pharmacol 2: 247-258.
7 Ambade A, Catalano D, Lim A, Kopoyan A, Shaffer SA, et al. (2014) Inhibition of heat shock protein 90 alleviates steatosis and macrophage activation in murine alcoholic liver injury. J Hepatol 4: 903-911.

8 Muralidharan S, Ambade A, Fulham MA, Deshpande J, Catalano D, et al. (2014) Moderate alcohol induces stress proteins HSF1 and hsp70 and inhibits proinflammatory cytokines resulting in endotoxin tolerance. J Immunol 193: 1975-1987.

9 Toth ME, Vigh L, Santha M (2014) Alcohol stress, membranes, and chaperones. Cell Stress Chaperones 3: 299-309.

10 Yang HY, Pu XP, Liu Y (2014). Chronic morphine treatment induces over-expression of HSP70 in mice striatum related with abnormal ubiquitin-proteasome degradation. Drug Alcohol Depend 139: 53-59.

11 Wang YT, Qin WJ, Liu Q, Li YL, Liang H, et al. (2014) Chaperone heat shock protein 70 in nucleus accumbens core: A novel biological target of behavioural sensitization to morphine in rats. Int $J$ Neuropsychopharmacol 3: 469-484.

12 Martinez-Laorden E, Hurle MA, Milanes MV, Laorden ML, Almela P (2012) Morphine withdrawal activates hypothalamic-pituitary-adrenal axis and heat shock protein 27 in the left ventricle: The role of extracellular signal-regulated kinase. J Pharmacol Exp Ther 3: 665-675. 